\title{
The deep project
}

\section{Evi Missouri-Khetab}

\section{Makarios III Hospital, Nicosia, Medical and Public Health Services, Cyprus}

\begin{abstract}
$\beta$ thalassaemia major is one of the most severe forms of anaemia. The recommended treatment consists in regular blood transfusions combined with chelating therapy to remove harmful iron accumulation in the body. The first chelating agent approved for clinical use was deferoxamine that, despite its potentially satisfactory therapeutic effects, is not usable by many patients due to its toxicity. Moreover, its subcutaneous route of administration leads to non compliance in most patients. On August 1999, an oral iron chelating agent, deferiprone, was authorised in Europe, and is currently indicated for the treatment of iron overload in patients with thalassaemia major when deferoxamine is contraindicated or inadequate. Despite a wide experience of deferiprone in thalassaemia patients, limited data are available on its use in children aged 2-10 years old and the need for additional data in this range of age is stated in the 2009 PDC0 (the Paediatric Committee at the European Medicinal Agency) priority list. In addition, the anticipated benefit in controlling cardiac iron overload in all paediatric population as well as in other chronic transfusion depend-
\end{abstract}

ent anaemias, lead the PDC0 to expand the request for additional data to the cited patients populations. The DEEP Project has been developed with the specific intent to integrate the existing information on deferiprone use in children aged 2-10 years old, thus covering the lack of knowledge and providing a valid support to the use of the drug in this age group. According to the recent scientific advancements and the opinion expressed by the PDCO, the project has been expanded to include the whole paediatric population and other chronic transfusion dependent anaemias. The aim of this application is to provide data on pharmacokinetics and comparative efficacy/safety of deferiprone, in order to produce an approved Paediatric Investigational Plan to be used for regulatory purposes. The trial is multicentric, open label and non-inferiority active- controlled. There are 12 participating centres from 9 different countries, including industry (Italy, UK, The Netherlands, Tunisia, Greece, Cyprus, Egypt and Canada). At the end of the proposed set of studies, deferiprone, will be available at efficacious dosages in children $<18$ years as first line treatment. In addition, a three year safety study will evaluate all deferiprone uses in the clinical setting.

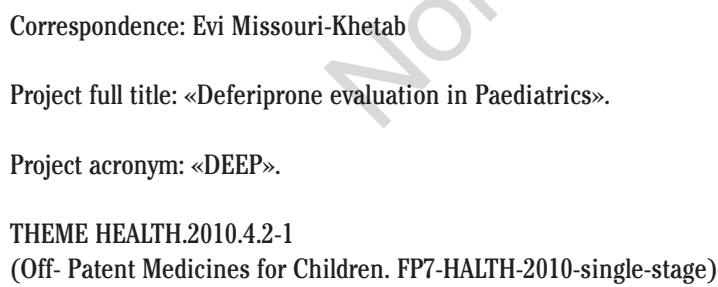

This article is distributed under the terms of the Creative Commons Attribution Noncommercial License (by-nc 3.0) which permits any noncommercial use, distribution, and reproduction in any medium, provided the original author(s) and source are credited.

Parts of this work were presented at the

" $3^{\text {rd }}$ Pan-European Conference on Haemoglobinopathies and Rare Anaemias", Limassol (Cyprus), 24-26 October 2012. 